\title{
Quantitative Measurement of Tool Embodiment for Virtual Reality Input Alternatives
}

\author{
Ayman Alzayat \\ University of Waterloo \\ Waterloo, Canada \\ aalzayat@uwaterloo.ca
}

\author{
Mark Hancock \\ University of Waterloo \\ Waterloo, Canada \\ mark.hancock@uwaterloo.ca
}

\author{
Miguel A. Nacenta \\ University of St Andrews \\ St Andrews, UK \\ mans@st-andrews.ac.uk
}

\begin{abstract}
Virtual reality (VR) strives to replicate the sensation of the physical environment by mimicking people's perceptions and experience of being elsewhere. These experiences are often mediated by the objects and tools we interact with in the virtual world (e.g., a controller). Evidence from psychology posits that when using the tool proficiently, it becomes embodied (i.e., an extension of one's body). There is little work, however, on how to measure this phenomenon in VR, and on how different types of tools and controllers can affect the experience of interaction. In this work, we leverage cognitive psychology and philosophy literature to construct the Locusof-Attention Index (LAI), a measure of tool embodiment. We designed and conducted a study that measures readiness-tohand and unreadiness-to-hand for three VR interaction techniques: hands, a physical tool, and a VR controller. The study shows that LAI can measure differences in embodiment with working and broken tools and that using the hand directly results in more embodiment than using controllers.
\end{abstract}

\section{CCS CONCEPTS}

-Human-centered computing $\rightarrow \mathbf{H C I}$ design and evaluation methods; Interaction devices; Empirical studies in HCI.

\section{KEYWORDS}

Embodiment, Tool Embodiment, Embodied Interaction, Virtual Reality, Ready-to-hand, Unready-to-hand, Tools.

\section{ACM Reference Format:}

Ayman Alzayat, Mark Hancock, and Miguel A. Nacenta. 2019. Quantitative Measurement of Tool Embodiment for Virtual Reality Input

Permission to make digital or hard copies of all or part of this work for personal or classroom use is granted without fee provided that copies are not made or distributed for profit or commercial advantage and that copies bear this notice and the full citation on the first page. Copyrights for components of this work owned by others than the author(s) must be honored. Abstracting with credit is permitted. To copy otherwise, or republish, to post on servers or to redistribute to lists, requires prior specific permission and/or a fee. Request permissions from permissions@acm.org. CHI 2019, May 4-9, 2019, Glasgow, Scotland, UK

(c) 2019 Copyright held by the owner/author(s). Publication rights licensed to ACM.

ACM ISBN 978-1-4503-5970-2/19/05_..\$15.00

https://doi.org/10.1145/3290605.3300673
Alternatives. In CHI Conference on Human Factors in Computing Systems Proceedings (CHI 2019), May 4-9, 2019, Glasgow, Scotland, UK. ACM, New York, NY, USA, 11 pages. https://doi.org/10.1145/3290605. 3300673

\section{INTRODUCTION}

Research in virtual reality has a long tradition striving to reproduce the sensations, perceptions and experience of being elsewhere (this is often referred to as presence [39, 43], immersion [19] or spatial-temporal deformation [1]). An important part of this presence is interaction; people in an immersive VR environment need to be able to interact realistically with the objects in the environment in order not to break the illusion of immersion in an alternate reality [37]. Presumably, in order to produce high-fidelity interaction in immersive VR environments, we also need an understanding of the issues of human physical interaction in the real world.

From research in psychology and philosophy, we know that interaction with objects of the environment is mediated by the physicality of our body $[8,9,11,29]$. I.e., interaction in this context is not only a matter of how an external object is perceived by the person's sensory mechanisms but, perhaps more importantly, involves how the human body itself affects the physical object and how the overall interplay between both the object and the body is processed by the brain.

To make things more complex, interaction with both physical and virtual objects is often mediated by tools. Tools are objects themselves that serve as intermediaries between our bodies and other objects that we intend to modify (e.g., we can use a stick in our hand to topple a vase that is out of our reach). Tools are particularly interesting in the context of human-computer interaction for two main reasons: first, VR interaction is often implemented through the use of input devices (e.g., the Oculus Touch VR Controller), which are themselves presented in VR as tools that mediate interaction with virtual objects. Second, from evidence in psychology and theories from philosophy, we know that tools become, in some ways, extensions of our own body when sufficient proficiency with the tool has been achieved. We know, for example, that experts often refer to their tools as "almost part of their own bodies", and that psychologists have found evidence that tool use can produce changes in body perception 
(people's body schemas are extended by the use of a tool [28]) and even extend the size of peripersonal space [7].

The influential philosopher Martin Heidegger discusses this kind of embodiment as a tool being "ready-to-hand" as opposed to "unready-to-hand" and "present-at-hand". A tool is "present-at-hand" when it is being examined (i.e., when it is considered as an object) and "ready-to-hand" when used in a way that it is not even noticed ("embodied"). "Unreadyto-hand" is an intermediate state, where the tool is broken, or the person is still developing sufficient skill with it.

In this paper we investigate an operationalization of these categories for measuring tool embodiment and apply it to measure three different interaction techniques in virtual environments: a physical tool, a VR controller, and the hand. We propose the use of the locus of attention index (LAI) as an indicator of tool embodiment, which measures whether attention is directed at the task or the tool before and after a tool is "broken". The study provides a validation of the measurement and a comparison of the tool embodiment levels of these three common ways to interact with virtual environments.

\section{RELATED WORK}

We review research concerning the areas of embodiment/ embodied interaction and measurements in virtual reality.

\section{Embodied interaction in $\mathrm{HCl}$ and related areas}

Embodied interaction is related to the ongoing discussion around NUIs [41] and TUIs [15]. These areas of HCI and UX relate to embodied interaction in that they both propose to bridge the physical properties of objects and the virtual presentation of information. Typical measurements in these areas are performance-based, used to evaluate input devices (e.g., [31]) or interaction techniques (e.g., [23]). Others in HCI have considered alternative measurements such as affect, task load, and motivation when using touch and tactile interaction $[36,40]$, and there exist specific measures of presence [43] and game immersion [6] that are useful to quantify subjective aspects of interface experiences. Although these methodologies use validated scales to measure the experience of using an interactive system, they do not directly address the issue of tool embodiment.

Influenced by the philosophical/epistemological investigations of Martin Heidegger, which were developed in "Sein und Zeit" (Being and Time) [11], Dourish [9], and Winograd \& Flores [42] also identify embodiment as a key concept in interaction design. Heidegger discusses three states of interacting with physical environments: ready-to-hand, unready-tohand, and present-at-hand. Ready-to-hand describes skilled action with a tool, for example using a functional tool to skilfully accomplish a task. Unready-to-hand describes a nonfunctional interaction with the world, such as when a tool is broken. Present-at-hand describes the user observing characteristics of the tool as a physical object. Both Dourish [9] and Winograd \& Flores [42], investigated these three states and applied them to characterize interactive systems. Dourish describes embodiment in a general sense that applies to people and objects when acting through a physical manifestation of the world [9, p. 100]. Winograd \& Flores [42] on the other hand, discuss how anticipation of breakdowns is essential in the design process, as it leads to change in attention to the different aspects of the interactive system, rather than accomplishing the task at hand.

More recently there have been attempts to quantify aspects of embodiment. Alzayat et al. [2] studied how physical objects and virtual objects presented on a multi-touch interactive surface manifest themselves in terms of the Kinesthetic Figural After-Effect (KFAE), a well-known perceptual phenomenon studied in Psychology [22]. They found that, unlike with physical objects, a multi-touch table simulating a physical object was not able to replicate this basic perceptual effect, therefore suggesting that experiences mediated through multi-touch interfaces might not be able to reproduce fundamental characteristics of physical interaction. In a follow up of their previous work, Alzayat et al. [3] address the issue of tool use and propose the use of attention measurements to compare a physical tool with a multi-touch counterpart. Although they found variations in the distribution of attention to the tool and to the task depending on the difficulty of the task, they did not find differences in the type of tool, and therefore their measurement cannot be used "as is" as a useful characterization of tool embodiment to compare different types of input. This paper builds upon Alzayat et al.'s idea of task-tool attention to propose a new index and measurement methodology that does show differences between input types and, importantly, tests the difference between ready-to-hand and unready-to-hand.

In addition to the KFAE there are other relevant phenomena in perception science that relate to embodiment but have not yet been successfully applied to quantify or discuss experience in interfaces. Examples include body schema [13], the rubber hand illusion [18, 25], and peripersonal space [7].

\section{Virtual reality}

Virtual reality (VR) is increasingly commonplace, and there is a steady advance in the availability and sophistication of its input/output mechanisms. VR and AR systems attempt to mimic the physical world by providing the same sensorimotor experiences of the physical environment [37]. Physiological responses and illusions that affect people in the physical environment can be reproduced in VR (e.g., public speaking anxiety [38] or a general sense of presence when navigating $[4,12,32-34])$. These exemplify how people's reactions in physical environments can be replicated virtually. 
Numerous studies have explored people's experience in VR with the goal of designing better systems that enhance interaction. These largely consist of self-report questionnaires, qualitative analysis or performance measures to evaluate the effectiveness of VR interaction (e.g., [10, 19, 20, 35]). These measures give information about the system's general usability and the impressions of immersion, but do not consider whether a particular tool or controls realistically reproduce interaction in the real world, much less tool embodiment.

Since VR offers the ability to manipulate the environment in ways that are impossible with physical reality, it has been used as a tool to investigate human cognition and perception. For example, one study used virtual reality to manipulate dimensions of people's fully-tracked virtual hands to investigate its influence on the perceived size and shape of virtual objects [24]. Participants provided a verbal estimate of the virtual object size. This experiment demonstrated increases in the perceived sizes of objects as a function of decreases in the size of one's virtual hand. Presumably, these influences are a result of using the body and its action capabilities as "perceptual ruler" to which perceived sizes and distances are scaled [24]. Another study [21] focused on the length of the arms in the virtual world using similar measures to the one used in rubber hand illusions, proprioceptive drifts. Although some of these paradigms are good potential approaches to measure and characterize interactions in VR they are difficult to implement to compare different input technologies. Specifically, when different virtual tools in the VR environment would not produce a proprioceptive drift similar to the rubber hand illusion.

\section{STUDY: MEASURING EMBODIED INTERACTION}

We conducted a study to validate an operationalization of a measure of tool embodiment based on the ready-to-hand and unready-to-hand concepts discussed by Heidegger [11], Dourish [9], and Winograd \& Flores [42]. As a secondary goal, we intended to learn about differences in tool embodiment between three common VR input alternatives: hand input, a VR controller, and physical props.

$\begin{gathered}\text { unready-to-hand } \\ \text { (tool broken) }\end{gathered}$
$\begin{gathered}\text { attention } \\ \text { to the tool }\end{gathered}$ $\begin{gathered}\begin{array}{c}\text { ready-to-hand } \\ \text { (tool working) }\end{array} \\ \text { distributed }\end{gathered}$

Figure 1: Our operationalization of tool embodiment relies on the idea that, when embodied with a tool, a person's attention will be on the task, rather than on the tool.

To operationalize tool embodiment, we leverage the idea that, when embodied with a tool (i.e., ready-to-hand), a person will be able to focus their attention on a task and will ignore the tool itself (Figure 1). When a tool breaks (i.e., unready-tohand), they will shift their attention to the tool. In our study, we ask participants to use one of three tools in an interactive task, and vary whether the tool works properly or breaks at fixed intervals. We then measure the difference in attention between working and broken states.

\section{Apparatus}

Participants were immersed in a virtual reality environment provided through Oculus Rift headset Model CV-1 and sensors to track the position of the headset. Participants sat at a $121.5 \mathrm{~cm}$ wide $\times 61 \mathrm{~cm}$ deep $\times 73 \mathrm{~cm}$ high table (see Figure 2) wearing the Oculus Rift and holding one of three controllers (see Conditions). The Oculus sensors and a tripod-mounted Kinect camera to detect hand and physical prop movements were placed on a table of the same height behind the first table, and the experimenter sat to the right side of the participant. The virtual environment was built with the Unity 3D game engine and programmed in C\#. All hardware and software were powered by a $3.5 \mathrm{GHz}$ computer with $16 \mathrm{~GB}$ of memory and a video card with a $1126 \mathrm{GHz}$ GPU and $4 \mathrm{~GB}$ of memory.

The virtual environment presented a table at the same height and position as in the physical environment, and a series of virtual objects to accomplish the task: a bookshelf with virtual cubes, placeholders for the cubes, and an information display (Figure 5a). The bookshelf contained a set of nine cubes in three rows, and each cube had a letter inscribed on it. Four placeholders for the cubes appeared on top of the table and in front of the information display. Each placeholder showed a target letter, and together spelled an English word. The information display showed instructions to the participant. All objects necessary for the task were designed to fit within the field of view of the participant.

\section{Tasks}

The individual trials were based on a dual-task paradigm [17]. The primary task was a simple puzzle in which participants had to use a hinged tool (Figure 3) to successively find and grab letter cubes from the bookshelf and place them (by releasing with the tool) in the placeholders marked with the corresponding letter. Participants were not bound by any order to place the letter cubes in the placeholders to complete a "word". When a word was completed, another set of cubes appeared and the cubes on the placeholders disappeared, revealing a new word to complete. The four-letter words were drawn randomly from a word bank of 1000 words; the set of nine cubes always included the four letters in the solution, with an additional five "distractor" cubes that had other letters. The details of how participants controlled the tool varied per condition and are therefore described in the Conditions section.

The secondary task consisted of noticing and reporting changes in colour of visual dots placed in two locations: on the cubes (which we call the task dots), and on the tool (the tool dots). There were two dots on each cube, located on its letter 


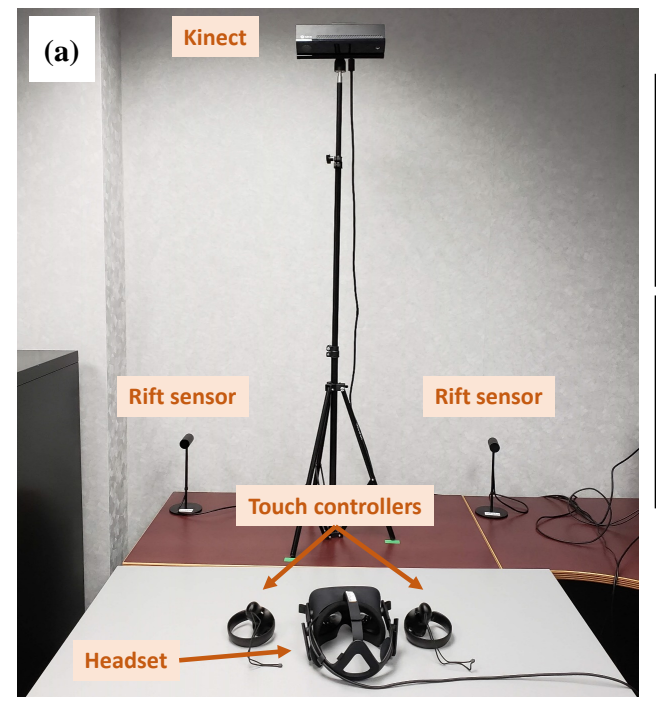

(b)

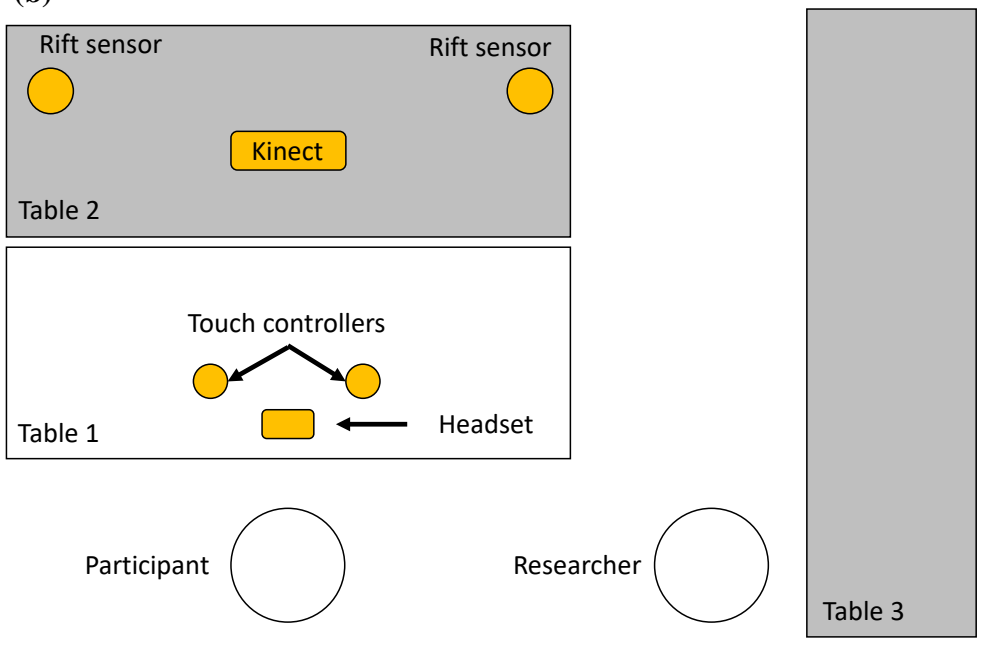

Figure 2: A photograph (a) and diagram (b) of the physical study setup.

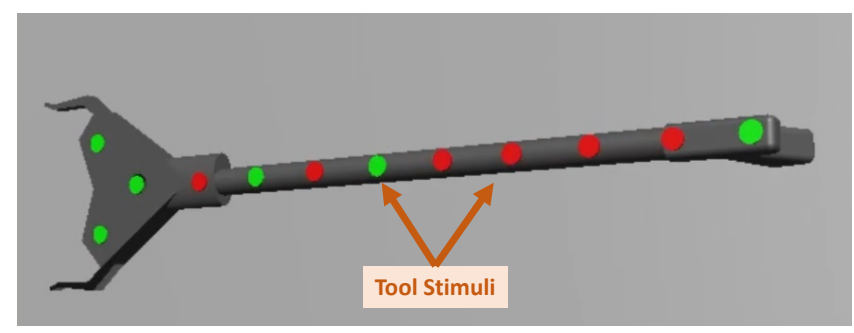

Figure 3: shows the virtual tool used in our study

side, for a total of 18 task dots. The location of the 12 tool dots is shown in Figure 5a. Each dot started randomly as either red or green, and one dot would change to the other colour every two seconds. Every dot changed exactly five times, but the order in which dots changed was random. Therefore, there were always exactly 150 changes during one 300 second trial ( 90 changes in task dots and 60 changes in tool dots). Participants were instructed to, while doing the primary task, pay attention to the dots and interrupt and pause the primary task by pressing a button with a controller in their non-dominant hand as soon as they noticed a dot change. When this happened, numbers appeared beside the dots and participants were asked to report verbally to the experimenter the number of the dot they saw change, which the experimenter recorded. After this, the numbers would disappear, and the primary task resumed.

\section{Conditions}

There were two main factors in the design of the experiment: the interaction technique for controlling the virtual tool (physical grabber, controller, or hand), and the state of the tool (working or broken). We describe first the three interaction techniques and then the two state conditions.Note that in all conditions the visual appearance and visibility of the virtual tool was identical (i.e., they always saw a grabber regardless of the control mechanism-Figure 3).

Physical Grabber. In the physical grabber condition, participants operated a grabber tool with their dominant hand. The dimensions of the grabber are $45.7 \mathrm{~cm} \times 10.2 \mathrm{~cm} \times 2.5 \mathrm{~cm}$. The dominant-hand Rift controller was attached to the middle of the grabber to track its position (the tool weighs $438 \mathrm{~g}$ with the controller attached, $136 \mathrm{~g}$ without). Additionally, two infrared reflective markers made the tips of the tool visible to the Kinect in order to detect the state of the tool (open or closed). Participants held the Rift's other controller on the non-dominant hand to initiate the secondary task by pressing the " $\mathrm{X}$ " button. Figure $4 \mathrm{c}$ shows the physical apparatus for this condition.

The grabber is operated by a trigger mechanism, resembling that of a pistol. This mechanism is continuous (i.e., gradual pulling of the trigger results in gradual closing of the hinge, until the tool's tips touch each other). The virtual and physical versions of the tool match in dimensions and position. Appearance is similar except that the virtual tool has additional area to accommodate some additional dots.

When grabbing a cube, the distance between the markers determined whether the cube was not yet grabbed (completely open to $50 \%$ aperture), grabbed and held (50\% aperture to $80 \%$ aperture), or dropped ( $80 \%$ aperture to completely closed). These thresholds were determined to resemble physical behaviour (closing the grabber too little does not hold a cube sufficiently firmly, and an excessively tight grip would result on the cube being squeezed out of the tool's tips). The physical tool has a spring mechanism (if the trigger is released the 

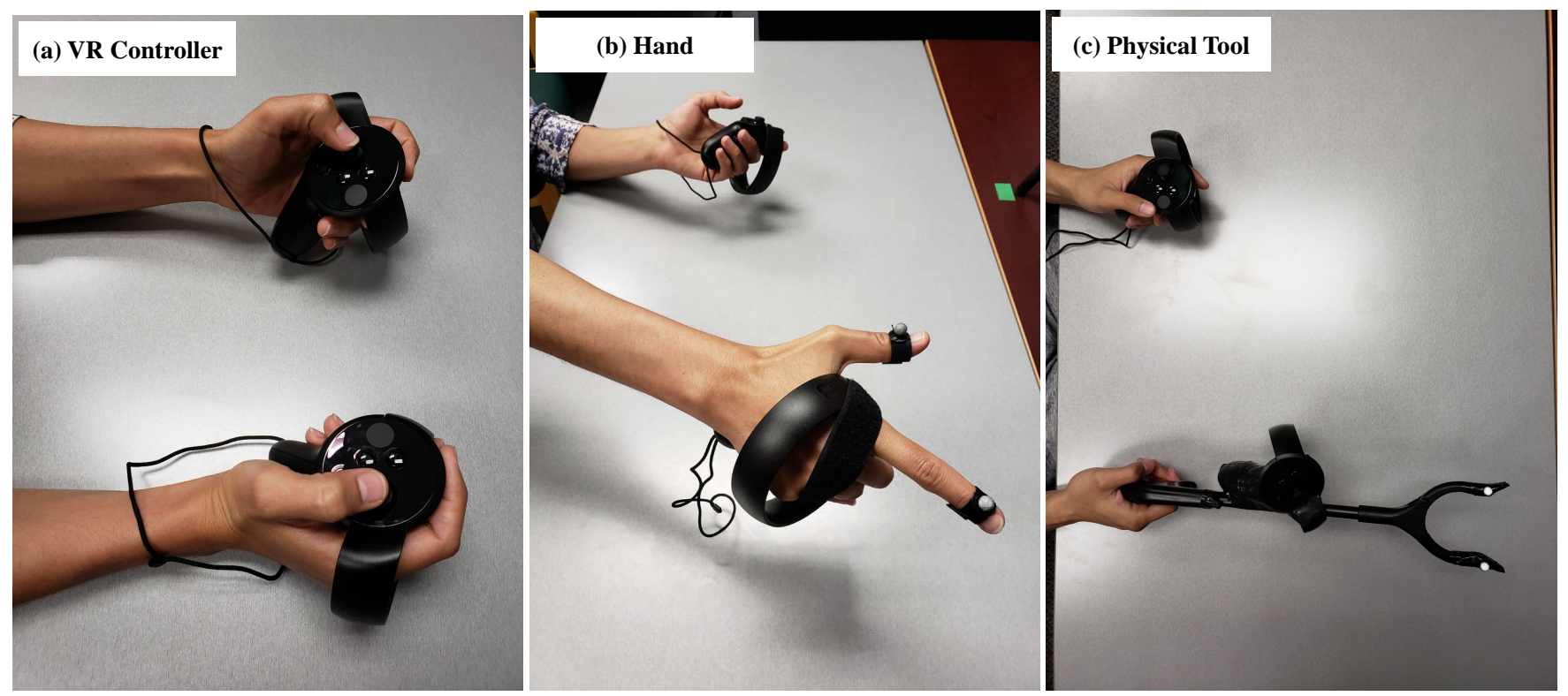

Figure 4: The three controllers used in our study.

hinge opens to the maximum aperture), but it does not have any interface with the VR system to simulate additional resistance on closing (it is just a tool with controller and markers attached for pose tracking).

Controller. The controller condition uses the Rift's left and right controllers in the manner they are designed to be held. The hinge of the tool is controlled by the dominant-hand controller's trigger mechanism, which is also continuous. The non-dominant hand works like in the physical tool condition (Figure $4 \mathrm{~b}$ ). The position of the controller in the physical world maps to the position of the handle of the virtual tool (not the tips). This is to maintain consistency in the range of participants' hand movements.

Hand. The hand condition required participants to use their own dominant hand to grab and release virtual cubes with their index finger and thumb. To track the hand we use the same controller, but attached with velcro to the hand to avoid having to hold it. The index and thumb fingers are tracked with reflective markers on their tips (as the grabber tool's tips above). The thresholds for cube holding were also the same as in the grabber condition and calibrated for aperture range (completely closed to completely open) at the beginning of each phase. As in the controllers condition, the hand position corresponds to the tool's handle (see also Limitations).

Working. The working condition is the normal state, where everything works as described above.

Broken. In the broken condition, the system simulates instances of tool failure at fixed intervals. There were two types of breakage: a pickup failure and a drop failure. In the case of a pickup failure, the participants would not be able to pick up the block, even if the aperture of the virtual grabber was within the appropriate threshold (i.e., the pressure applied to the tool or controller trigger, or the distance between the two fingers of the hand was correct). Participants had to press the trigger a number of times to be able to pick up the block. In the case of a drop failure, as the participant was moving a block from the bookshelf to the placeholder, the system would cause the block to drop, even if the aperture of the virtual grabber was correct (Figure 6). The participant was then required to pick up the cube again to continue. Pick up failure operated with the following sequence: no pick up, pick up, no pick up. In the drop failure the cube would drop with the following sequence drop, no drop, drop.

Note that participants were not aware that the tool was designed to break in the broken condition. This small but necessary deception was addressed and approved by our ethics review board.

\section{Participants \& Procedure}

Sixty participants (32 female, 27 male, 1 undisclosed gender, ages 16-46, $M d n=23$ ) were recruited via on-campus mailing lists from the local university. Four additional participants took part, but their data were excluded from the analysis due to technical difficulties with the VR apparatus. These difficulties occurred at the beginning of the experiment and included some bugs in the game that were corrected. Participants were screened for handedness (all self-reported to be right-handed), colour-blindness (all passed the Ishihara Color 

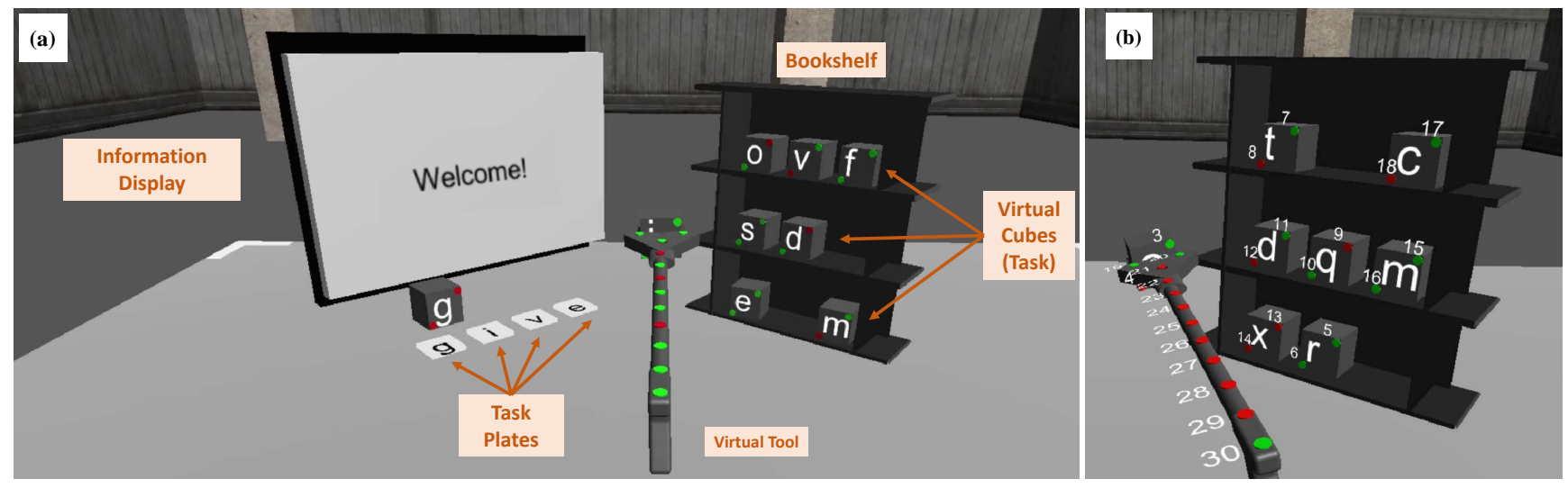

Figure 5: Virtual study Scene Setup. a. Virtual study scene setup with a virtual tool, bookshelf, task plates, and information display. $b$. The virtual study scene when the pause button is pressed. Numbers on visual dots support the secondary task.

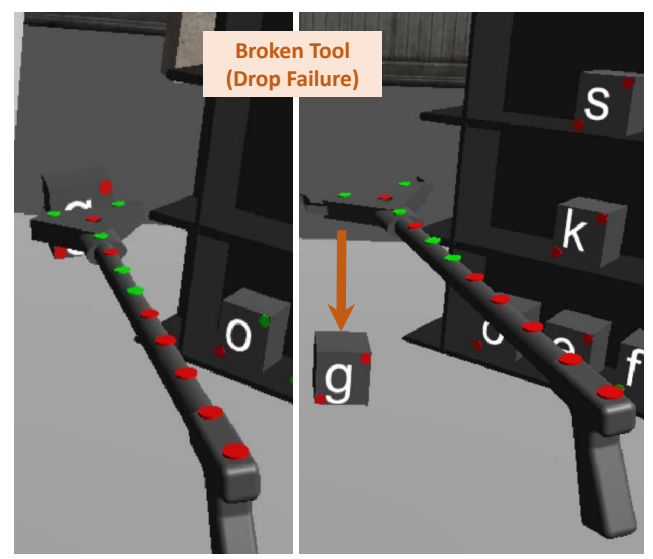

Figure 6: From left to right, example of breakage (cube falls).

Blindness Test [14]). Participants received \$15 gift certificates for participating.

The study lasted approximately $1.5 \mathrm{~h}$ and used a mixed design: between-participants for the interaction technique factor (grabber, controllers, hand), and within-participants for the tool state factor (working or broken). Participants were randomly assigned an interaction technique, and the order of tool state was counterbalanced (i.e., for each interaction technique, half of the participants started with a working tool, and half with a broken tool). Although we considered a within-participants design for both factors, we eventually chose this mixed design because our pilots showed a strong learning effect between conditions and, more importantly, because a fully within-participants design would have required over $4 \mathrm{~h}$ to complete for each participant.

Participants first gave written consent, completed the Ishihara test, and underwent two phases of training. In the first training phase, they familiarized themselves with the VR environment by moving around the cubes (primary task) without the need to report when dots changed colour using a working tool. In the second training phase, participants only practiced on reporting any changes in the dots colour to familiarize themselves with the reporting task, interaction technique, and the mechanics of pausing to report colour changes.

The core of the experiment consisted of four trials, alternating between working and broken two times in the same counterbalanced order. Each trial lasted exactly 300 seconds, in which participants would complete a variable number of puzzle task words but were always shown 150 changes. At the end of each trial, participants had a short break of approximately five minutes, during which they also completed questionnaires about the previous trial.

\section{Measurements}

Here we describe the main measurements taken during the trials. There are measurements of attention, performance and engagement/presence.

Measures of Attention. We measured the number of noticed changes for each dot and grouped them by whether they were on the tool (60 changes) or on the cubes (90 changes). Since these totals did not match, we instead normalized the noticed changes in each category as detection rate (DR):

$$
\begin{aligned}
D R_{\text {tool }} & =\frac{\# \text { noticed changes on tool }}{60} \\
D R_{\text {task }} & =\frac{\# \text { noticed changes on cubes }}{90}
\end{aligned}
$$

Locus of Attention Index. Rather than focusing on the base detection rates, we were more interested in the relationship between $D R_{\text {task }}$ and $D R_{\text {tool }}$, since our main interest is in providing an operationalization of the concepts of ready-to-hand and unready-to-hand. For instance, in a ready-to-hand state, we would expect detection rates to be higher at the task than on the tool, and vice versa for unready-to-hand states. We 
therefore propose the Locus of Attention Index (LAI), calculated as follows:

$$
L A I=\frac{D R_{\text {task }}-D R_{\text {tool }}}{D R_{\text {task }}+D R_{\text {tool }}},
$$

We obtain an LAI for each trial, resulting in four different LAI measurements per participant, two for each of the tool state conditions (working and broken). Notice that the LAI varies between +1 (all changes detected are in the task) and -1 (all changes detected are on the tool), with zero indicating that detected changes are equally distributed between the task and tool (Figure 7). The LAI ignores the base detection rates; that is, it obviates whether the detection rates are low or high (which could vary significantly between individuals) and focuses on which area (task or tool) has the dominant detection rate. Note that the term "locus" refers to where the attention is spatially, and not temporally. LAI is a cumulative measure across the entirety of a trial, not a real-time metric.

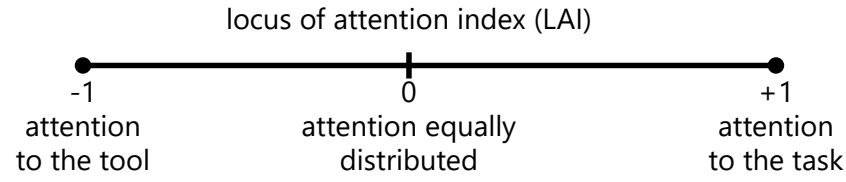

Figure 7: Our LAI measure corresponds to our theory above and measures across the entirety of a trial whether the attention is on the tool (negative) or on the task (positive).

Shift in Locus of Attention Index. The last measure of attention, which is also the measure at the highest level of abstraction, is the shift in LAI (SLAI) between two consecutive trials (working to broken, or broken to working):

$$
\begin{aligned}
& S L A I_{W-B}=L A I_{\text {broken }}-L A I_{\text {working }} \\
& S L A I_{B-W}=L A I_{\text {working }}-L A I_{\text {broken }}
\end{aligned}
$$

This second-order measurement intends to record, for a given interaction technique, how much changing the state of a tool (i.e., "breaking" or "fixing" it) affects the locus of attention. For example, a negative $S L A I_{W-B}$ (i.e., a decreasing $L A I$ ) would indicate that changing from a working to broken tool corresponds to detecting more changes on the tool, which may indicate a shift from ready-to-hand to unready-to-hand.

Depending on the order the participant has been assigned, they might have two measures of one and one of the other (e.g., a participant that did $W-B-W-B$ would have two $S L A I_{W-B}$ measures and one $S L A I_{B-W}$ measure). For simplicity, we take only the first two transitions.

Performance, Engagement and Presence. In addition to our measures of attention, we measured the number of words completed, which is inversely proportional to the time taken per word. Additionally, participants completed the Game Engagement Questionnaire (GEQ, [6]), which consists of 19 questions

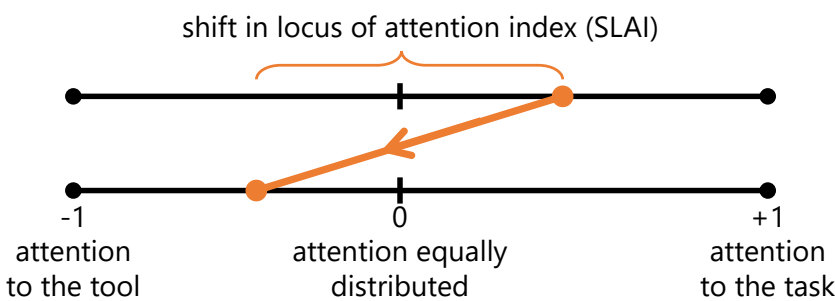

Figure 8: SLAI measures a shift in attention from either the task to the tool (e.g., when a tool breaks) or vice versa.

that produce a score between 19 to 57, where an increase in the GEQ scores represents more engagement with the game tested. Participants also completed the realism part of the Presence Questionnaire (PQ, [43]), which consists of seven 7-point Likert scale questions, with higher values indicating a more realistic experience.

\section{Hypotheses}

In general, we expected the LAI measure to show differences between the working and broken conditions, as a reflection of the concepts of ready- and unready-to-hand. We were also interested in the comparison of LAI levels between the different devices. We expected that the physical grabber condition would show generally higher levels of LAI than the controllers, since it corresponds better with the virtual tool. We were not sure what to expect regarding the hand, since this could go both ways: if what is important for tool embodiment is that the VR visuals correspond to the physical reality, then we could expect LAI to be lower for the hand. If the LAI provides a measurement of how embodied we are with the tool, we would expect the hand to have the highest levels, since one cannot be more embodied than with their own limb. We also expected that the second-order measurement (SLAI) would become more pronounced (i.e., have a higher magnitude) for interaction techniques with higher LAIs. In other words, if one is more embodied with a tool, it should become more apparent when the tool "breaks". Finally, we expected the game engagement questionnaire and the presence questionnaire to follow roughly the same patterns as LAI, with higher engagement and presence when the tool is working, and different values for the different interaction techniques. However, due to the betweenparticipants nature of the experiment we would not be able to calculate correlations between the attention and questionnaire measures. The main hypotheses are encoded as follows:

H1. LAI will be higher when the tool is working than when it is broken.

H2. The levels of LAI will be different for different interaction techniques.

H3. The physical grabber will have a higher LAI than the controller. 


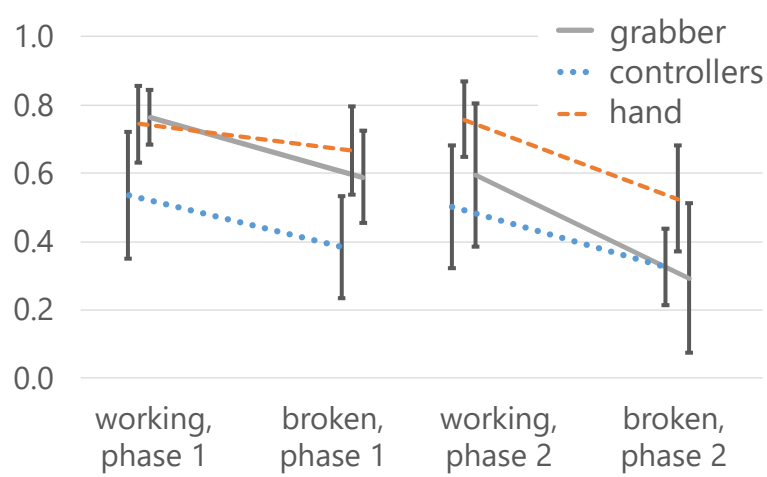

Figure 9: Results for the locus of attention index (LAI). There were main effects of interaction technique, state of the tool (working/broken) and phase. Note only $[0,1]$ range of possible $[-1,1]$ range is represented.

H4. Shifts in the locus of attention between working and broken conditions (SLAI) will be different for different interaction techniques.

H5. Levels of game engagement (H5a) and presence (H5b) will be higher when the tool is working than when broken.

H6. Levels of game engagement (H6a) and presence (H6b) will be different for the different interaction techniques.

\section{RESULTS}

We address first the analysis of the data regarding the LAI (H1-H3), as well as the absolute measurement of changes noticed. We then look at the shift of LAI, and the GEQ and PQ measurements (H4-H6). Finally, we include a section on nonplanned or statistically-untestable observations. All analysis was run on IBM SPSS v.24. All post-hoc comparisons between tools are corrected for multiple comparisons with Scheffe's procedure [5], whenever the sphericity assumption is broken we apply Greenhouse-Geisser's correction. Error bars in charts indicate $95 \%$ confidence intervals.

\section{Locus of Attention Index Analysis}

We ran a mixed design repeated measures ANOVA with interaction technique (grabber, controllers, hand) as a betweenparticipants factors and state (working, broken) and phase $(1,2)$ as within-participant factors on our LAI measure. The ANOVA showed a main effect of the state factor $\left(F_{1,57}=20.52\right.$, $p<.001, \eta_{p}^{2}=.27$ ). LAIs are lower (more attention on the tool) for the broken condition $\left(M_{B}=.46, S E_{B}=0.03 ; M_{W}=.65\right.$, $\left.S E_{W}=0.04\right)$. Therefore, $\mathrm{H} 1$ is supported.

The ANOVA showed a main effect of interaction technique $\left(F_{2,57}=4.32, p=.018, \eta_{p}^{2}=.13\right)$, supporting H2. Post-hoc analysis showed that using hands was statistically different than using controllers $(p=.018)$, but the other two comparisons were not significant $(p>.05)$. The average LAIs of the three input conditions (across both state conditions) were highest for hand $(M=.67, S E=.057)$ and lowest for controllers $(M=.44$, $S E=.057)$, with the grabber in between $(M=.56, S E=.057)$. $\mathrm{H} 3$ is therefore not supported, although also not contradicted by the results of the analysis. Instead, if we interpret LAI as a measure of tool embodiment, hand seems to be the most embodied type of input, and controllers the least, with the grabber somewhere in the middle.

There was also a significant main effect of phase $\left(F_{1,57}=\right.$ $\left.8.51, p<.005, \eta_{p}^{2}=.13\right)$, with LAIs being higher in the first phase $(M=.61, S E=0.03)$ than the second $(M=.50, S E=0.04)$. Since none of the other two-way or three-way interactions were significant we do not perform a separate analysis per phase. Figure 9 shows the distribution of the indices split by interaction technique, state and phase.

\section{Shift of Locus of Attention Index Analysis}

To discern whether the SLAI measurements can be a useful measure we calculated two SLAI values (the first working-tobroken and the first broken-to-working) for each participant and ran two ANOVAs with interaction technique and order as between-participants factors. Although the average values are consistent with our expectations $\left(S L A I_{W-B}\right.$ is negative on average and $S L A I B-W$ is positive), neither of the two tests showed any significant main effects or interactions, leaving H4 without support.

\section{GEQ/Presence Questionnaire Analysis}

We ran the same ANOVA as in the LAI analysis with GEQ as the dependent variable. The only significant main effect was state $\left(F_{1,57}=17.00, p<.001, \eta_{p}^{2}=.23\right)$, which supports H5a. There was a marginally significant main effect of interaction technique $\left(F_{2,57}=2.85, p<.066, \eta_{p}^{2}=.09\right)$, which means that there is not support for H6a. Figure 10 shows the distributions of the GEQ scores by technique, state and phase. Similarly, the only significant main effect for the PQ dependent variable was state $\left(F_{1,57}=83.66, p<.001, \eta_{p}^{2}=.60\right)$ (H5b supported), with none of the other tests being significant (H6b not supported).

\section{Performance measurements}

To complete the analysis, we ran the same ANOVA as in the LAI analysis on our performance measure (the count of words that participants were able to complete in the primary task). As expected, there was a significant effect of state $\left(F_{1,57}=758.89\right.$, $\left.p<.001, \eta_{p}^{2}=.93\right)$ and phase $\left(F_{1,57}=34.74, p<.001, \eta_{p}^{2}=.38\right)$, showing that people perform better when the tool is working and in later phases. There was also an interaction between state and phase $\left(F_{1,57}=29.40, p<.006, \eta_{p}^{2}=.13\right)$ that is not of interest to us. 


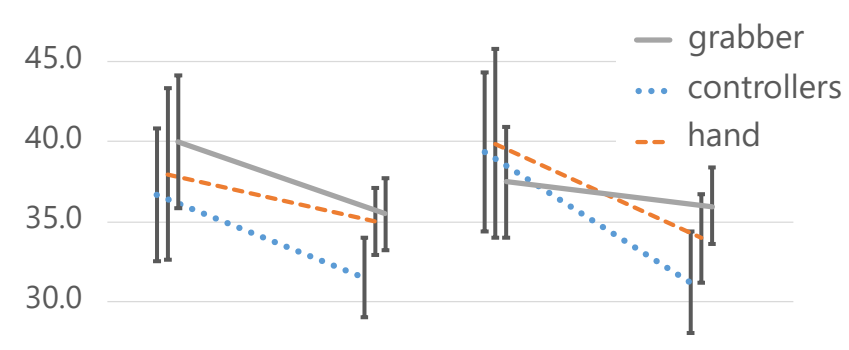

25.0
working
broken,
working,
broken,
phase 1
phase 1
phase 2
phase 2

Figure 10: Results for engagement (GEQ). There was a significant main effect of state (working/broken) and a marginally significant effect of interaction technique.

More importantly, the main effect of interaction technique was not significant $\left(F_{1,57}=2.06, p<.137, \eta_{p}^{2}=.067\right)$, which suggests that, if the other measurements showed some ability to discriminate between techniques, these differences cannot be explained by performance measures alone; in fact, mean performance values are fairly close and do not have the same order as the LAI measurements (hand: $M=19.25$ words, $S E=0.95 \mathrm{w}$; controllers: $M=17.56 \mathrm{w}, S E=0.95 \mathrm{w}$; grabber: $M=16.56 \mathrm{w}, S E=0.95 \mathrm{w})$.

\section{Other Observations}

We describe in this section several observations and unplanned analyses because they might provide interesting insight and hints for further research, but readers should be careful not to over-interpret these findings, since the study was not designed to answer these questions.

The first observation is that estimated standard deviations of the LAI, GEQ and PQ measures are, for the most part, much larger in the second phase than in the first phase. When this is not the case they are roughly equivalent, except for two cases (see Table 1). Although we cannot provide a statistically meaningful test for the differences in variance (we only have one variance estimate across all participants), this observation suggests that the first phase of testing might be less noisy, and therefore, more informative, than the second.

Due to the general increase in variance, and although the phase $\times$ technique interaction was not significant for LAI, GEQ or PQ in the ANOVAs above $\left(F_{2,57}=\{2.342, .732,1.666\}\right.$, $p=\{.11,49, .20\}$, respectively), we decided to run ANOVAS with only the first phase data, to see if we could better discriminate between the different interaction techniques.

Despite the loss in power due to the reduction in data points, these ANOVAs result in a significant main effect for both the LAI and the GEQ measurement $\left(F_{2,57}=\{5.76,3.80\}\right.$, $\left.p=\{.005, .028\}, \eta_{p}^{2}=\{.168, .118\}\right)$. For the LAI measurement,

\begin{tabular}{|c|c|c|c|c|c|}
\hline & Input & State & $\begin{array}{c}\text { SD } \\
\text { (Ph. 1) }\end{array}$ & $\begin{array}{c}\text { SD } \\
(\mathbf{P h} .2)\end{array}$ & Inc.\% \\
\hline \multirow{6}{*}{ LAI } & \multirow{2}{*}{ Grabber } & $\mathrm{W}$ & 0.18 & 0.48 & 160 \\
\hline & & B & 0.31 & 0.50 & 61 \\
\hline & \multirow{2}{*}{ Controllers } & W & 0.42 & 0.41 & -3 \\
\hline & & B & 0.34 & 0.26 & -25 \\
\hline & \multirow{2}{*}{ Hand } & W & 0.26 & 0.25 & -3 \\
\hline & & B & 0.30 & 0.36 & 20 \\
\hline \multirow{6}{*}{ GEQ } & \multirow{2}{*}{ Grabber } & W & 5.11 & 5.46 & 7 \\
\hline & & $\mathrm{B}$ & 5.03 & 6.42 & 28 \\
\hline & \multirow{2}{*}{ Controllers } & W & 5.76 & 7.25 & 26 \\
\hline & & $\mathrm{B}$ & 6.61 & 8.45 & 28 \\
\hline & \multirow{2}{*}{ Hand } & W & 4.87 & 6.34 & 30 \\
\hline & & B & 4.45 & 5.98 & 34 \\
\hline \multirow{6}{*}{$\mathbf{P Q}$} & \multirow{2}{*}{ Grabber } & W & 5.97 & 6.13 & 3 \\
\hline & & B & 9.41 & 7.89 & -16 \\
\hline & \multirow{2}{*}{ Controllers } & $\bar{W}$ & 9.68 & 11.98 & 24 \\
\hline & & B & 9.43 & 11.34 & 20 \\
\hline & \multirow{2}{*}{ Hand } & $\overline{\mathrm{W}}$ & 8.03 & 9.97 & 24 \\
\hline & & B & 12.185 & 13.453 & 10 \\
\hline
\end{tabular}

Table 1: Standard deviations for the LAI, GEQ, and PQ measures in our study. $S D$ s are larger in the second phase than the first (\% increase in right-most column).

the post-hocs between input conditions are now, in addition to the previous discrimination between hand and controllers $(p=.012)$, also significant between the grabber and controllers $(p=.03)$. For the GEQ there is now a statistically significant difference between the grabber and controllers, whereas there was no difference before.

\section{GENERAL DISCUSSION}

This work presents evidence of Heidegger's readiness-tohand and unreadiness-to-hand in a VR environment with three different controllers. Here is a summary of our findings:

- The LAI measure was an effective way to measure embodiment-LAI was higher when the tool was working and lower when it was broken. This supports H1, indicating that, in the working condition, people pay more attention to the task and less to the tool, but in the broken condition, attention partially shifts from the task to the tool.

- Different interaction techniques had different LAI values, which supports $\mathrm{H} 2$.

- The hand had the highest LAI, then the grabber, and then the controller; however only the hand and controller were significantly different, and therefore $\mathrm{H} 3$ was not supported.

- Our second-order SLAI measure did not reveal any significant differences, therefore $\mathrm{H} 4$ was not supported. 
- The GEQ/presence questionnaires showed higher engagement/presence when participants used working tools than when they were broken, confirming $\mathrm{H} 5 \mathrm{a}$ and $\mathrm{H} 5 \mathrm{~b}$.

- While our performance measure (number of words completed) revealed, unsurprisingly, that participants performed better with working tools than broken ones and improved performance between phases, it could not discriminate between interaction techniques, indicating that our LAI measure provides a more nuanced understanding than performance measures alone.

Our study provides the first empirical investigation to support Heidegger's phenomenon of readiness-to-hand and unreadiness-to-hand using different interaction techniques in virtual reality. In particular, LAI can be used as a measure of tool embodiment showing different levels of the tool "disappearing" (i.e., attention shifting to the task rather than the tool) when a participant is engaged in the task.

Notably, the LAI measure was higher with hand input than with the controller, indicating that hand interaction may be more embodied than current VR controllers (though this finding cannot currently be generalized beyond our specific designs for these techniques). More specifically, participants were able to pay more attention to their task when using hand interaction than when using Oculus controllers, despite there being no observable performance difference.

\section{Implications of using LAI for $\mathrm{HCI}$}

The LAI measure provides a more direct method for measuring embodied interaction for interactive technologies. In our study, we used a controller, a hand, and a grabber in the physical world to control virtual a tool. The virtual tool and objects used in the study were identical in terms of the visual stimuli presented. This technique would allow other interactive technologies to use LAI to measure tool embodiment and embodied interaction. Our work can be applied as a measure provided these elements exists in the experimental setup:

- A tool: to measure tool embodiment, there must be some mediator between a person's body and the artifacts they are interacting with. This can be a virtual or physical tool.

- Dual-task paradigm: future experimenters must determine a primary task to complete with the interactive tool, and then can use our secondary task of detecting changes. In our study, this was a change of colour, but to calculate LAI, there need only be a sequence of changes of any kind.

- Tool breakage: an important aspect of the LAI is having both working and broken states. It must be possible to have the tool malfunction in some controlled way.

The combination of these elements provides the necessary information to calculate LAI and begin to draw conclusions about a tool's level of embodiment.

\section{Limitations and Future Work}

Although we provide the first account of quantitatively measuring tool embodiment in VR, we present data for only three common interaction techniques. Future work could adopt our findings with other interactive systems like mid-air gestures. Furthermore, by combining the LAI measure with eyetracking, it may be possible to create a live "tool embodiment" indicator. Eye-tracking alone might not be a strong indicator of selective attention, since looking does not always equate to attention, for example due to change blindness [26], inattentional blindness [16, 27], covert attention [30] and the variation of location within the fovea.

Note also that our choice to map hand position to the handle of the virtual tool in the controllers and hands conditions (to avoid systematic differences in motor behavior) could potentially (although not likely, in our opinion) have had an effect on measurements. This requires further experimentation that can help determine, at a finer granularity, the main factors in the experience of tool embodiment.

\section{CONCLUSION}

This paper presented a study that measures readiness-tohand and unreadiness-to-hand with different interaction techniques (hand, controller, and grabber) in virtual reality. We introduced a novel measure, the Locus of Attention Index (LAI), that indicates the level of tool embodiment when using a tool for a specific task. Results show that our LAI measure is higher in ready-to-hand situations and lower in unreadyto-hand situations. Finally, we discuss how the LAI can be used with other interactive technologies as a measurement of embodied interaction.

\section{ACKNOWLEDGMENTS}

Thanks to Licheng Zhang for helping to build the experimental software, our participants, and the anonymous reviewers. We also thank the Ontario Ministry of Research and Innovation and Science and the Natural Sciences and Engineering Research Council of Canada for funding.

\section{REFERENCES}

[1] Merwan Achibet, Adrien Girard, Anthony Talvas, Maud Marchal, and Anatole Lécuyer. 2015. Elastic-Arm: Human-scale passive haptic feedback for augmenting interaction and perception in virtual environments. In Virtual Reality (VR), 2015 IEEE. IEEE, IEEE, Arles, France, 63-68.

[2] Ayman Alzayat, Mark Hancock, and Miguel Nacenta. 2014. Quantitative Measurement of Virtual vs. Physical Object Embodiment Through Kinesthetic Figural After Effects. In Proceedings of the SIGCHI Conference on Human Factors in Computing Systems (CHI '14). ACM, New York, NY, USA, 2903-2912. https://doi.org/10.1145/2556288.2557282

[3] Ayman Alzayat, Mark Hancock, and Miguel A. Nacenta. 2017. Measuring Readiness-to-Hand Through Differences in Attention to the Task vs. Attention to the Tool. In Proceedings of the 2017 ACM International Conference on Interactive Surfaces and Spaces (ISS '17). ACM, New York, NY, USA, 42-51. https://doi.org/10.1145/3132272.3134114 
[4] Woodrow Barfield and Suzanne Weghorst. 1993. The sense of presence within virtual environments: A conceptual framework. Advances in Human Factors Ergonomics 19 (1993), 699-699.

[5] Robert Bohrer. 1967. On sharpening Scheffe bounds. Fournal of the Royal Statistical Society. Series B (Methodological) 29 (1967), 110-114.

[6] Jeanne H Brockmyer, Christine M Fox, Kathleen A Curtiss, Evan McBroom, Kimberly M Burkhart, and Jacquelyn N Pidruzny. 2009. The development of the Game Engagement Questionnaire: A measure of engagement in video game-playing. Journal of Experimental Social Psychology 45, 4 (2009), 624-634.

[7] Lucilla Cardinali, Francesca Frassinetti, Claudio Brozzoli, Christian Urquizar, Alice C Roy, and Alessandro Farnè. 2009. Tool-use induces morphological updating of the body schema. Current Biology 19, 12 (2009), R478-R479.

[8] Andy Clark. 2008. Supersizing the mind: Embodiment, action, and cognitive extension. OUP USA, New York, USA.

[9] Paul Dourish. 2004. Where the action is: the foundations of embodied interaction. MIT press, Cambridge.

[10] Dalma Geszten, Balázs Péter Hámornik, Anita Komlodi, Károly Hercegfi, Bálint Szabó, and Alyson Young. 2015. Qualitative analysis of user experience in a 3D virtual environment. In Proceedings of the 78th ASIS\& T Annual Meeting: Information Science with Impact: Research in and for the Community (2015). American Society for Information Science, Silver Springs, MD, USA, 124.

[11] Martin Heidegger. 1996. Being and time: A translation of Sein und Zeit. SUNY press, New York, USA.

[12] Richard M Held and Nathaniel I Durlach. 1992. Telepresence. fournal of communication 1, 1 (1992), 109-112.

[13] Nicholas P Holmes and Charles Spence. 2006. Beyond the body schema: Visual, prosthetic, and technological contributions to bodily perception and awareness. Human body perception from the inside out 1 (2006), $15-64$.

[14] Shinobu Ishihara. 1918. Tests for Color Blindness. Medical Record 1, 5 (1918), 376.

[15] Hiroshi Ishii. 2008. The tangible user interface and its evolution. Commun. ACM 51, 6 (2008), 32-36.

[16] Melinda S Jensen, Richard Yao, Whitney N Street, and Daniel J Simons. 2011. Change blindness and inattentional blindness. Wiley Interdisciplinary Reviews: Cognitive Science 2, 5 (2011), 529-546.

[17] Daniel Kahneman. 1973. Attention and effort. Vol. 1063. Citeseer, USA.

[18] MPM Kammers, Frederique de Vignemont, Lennart Verhagen, and $\mathrm{H}$ Chris Dijkerman. 2009. The rubber hand illusion in action. Neuropsychologia 47, 1 (2009), 204-211.

[19] Shunichi Kasahara, Keina Konno, Richi Owaki, Tsubasa Nishi, Akiko Takeshita, Takayuki Ito, Shoko Kasuga, and Junichi Ushiba. 2017. Malleable embodiment: Changing sense of embodiment by spatial-temporal deformation of virtual human body. In Proceedings of the 2017 CHI Conference on Human Factors in Computing Systems (2017) (CHI '17). ACM, New York, NY, USA, 6438-6448. https://doi.org/10.1145/3025453.3025962

[20] Konstantina Kilteni, Raphaela Groten, and Mel Slater. 2012. The sense of embodiment in virtual reality. Presence: Teleoperators and Virtual Environments 21, 4 (2012), 373-387.

[21] Konstantina Kilteni, Jean-Marie Normand, Maria V Sanchez-Vives, and Mel Slater. 2012. Extending body space in immersive virtual reality: a very long arm illusion. PloS one 7, 7 (2012), e40867.

[22] Wolfgang Köhler and Dorothy Dinnerstein. 1947. Figural after-effects in kinesthesis. Miscellanea psychologica Albert Michotte 199 (1947), 196-220.

[23] Daniel Leithinger and Michael Haller. 2007. Improving menu interaction for cluttered tabletop setups with user-drawn path menus. In Horizontal Interactive Human-Computer Systems, 2007. TABLETOP'07.
Second Annual IEEE International Workshop on (2007). IEEE, Newport, RI, USA, 121-128.

[24] Sally A Linkenauger, Markus Leyrer, Heinrich H Bülthoff, and Betty J Mohler. 2013. Welcome to wonderland: The influence of the size and shape of a virtual hand on the perceived size and shape of virtual objects. PloS one 8,7 (2013), e68594.

[25] Matthew R Longo, Friederike Schüür, Marjolein PM Kammers, Manos Tsakiris, and Patrick Haggard. 2008. What is embodiment? A psychometric approach. Cognition 107, 3 (2008), 978-998.

[26] Li-Qian Ma, Bi-Ye Jiang, Shi-Min Hu, Kun Xu, and Tien-Tsin Wong. 2013. Change blindness images. IEEE transactions on visualization and computer graphics 19, 11 (2013), 1.

[27] Arien Mack. 2003. Inattentional blindness: Looking without seeing. Current Directions in Psychological Science 12, 5 (2003), 180-184.

[28] Paul M McDonnell, Robert N Scott, Jacqueline Dickison, Rose Anne Theriault, and Bradley Wood. 1989. Do artificial limbs become part of the user? New evidence. Fournal of rehabilitation research and development 26, 2 (1989), 17-24.

[29] Alva Noë. 2004. Action in perception. MIT press, london, England.

[30] Michael I Posner. 1980. Orienting of attention. Quarterly journal of experimental psychology 32, 1 (1980), 3-25.

[31] Kathy Ryall, Clifton Forlines, Chia Shen, and Meredith Ringel Morris. 2004. Exploring the effects of group size and table size on interactions with tabletop shared-display groupware. In Proceedings of the 2004 ACM conference on Computer supported cooperative work (2004). ACM, New York, NY, USA, 284-293.

[32] Maria VSanchez-Vives and Mel Slater. 2005. From presence to consciousness through virtual reality. Nature Reviews Neuroscience 6, 4 (2005), 332.

[33] Thomas B Sheridan. 1992. Telerobotics, automation, and human supervisory control. MIT press, london, England.

[34] Thomas B Sheridan. 1996. Further musings on the psychophysics of presence. Presence: Teleoperators \& Virtual Environments 5, 2(1996), 241-246.

[35] Roland Sigrist, Georg Rauter, Robert Riener, and Peter Wolf. 2013. Augmented visual, auditory, haptic, and multimodal feedback in motor learning: a review. Psychonomic bulletin \& review 20, 1 (2013), 21-53.

[36] Daniel J Simons and Daniel T Levin. 1998. Failure to detect changes to people during a real-world interaction. Psychonomic Bulletin \& Review 5, 4 (1998), 644-649.

[37] Mel Slater. 2009. Place illusion and plausibility can lead to realistic behaviour in immersive virtual environments. Philosophical Transactions of the Royal Society of London B: Biological Sciences 364, 1535 (2009), 3549-3557.

[38] Mel Slater, David-Paul Pertaub, Chris Barker, and David M Clark. 2006. An experimental study on fear of public speaking using a virtual environment. CyberPsychology \& Behavior 9, 5 (2006), 627-633.

[39] Anthony Steed, Sebastian Frlston, Maria Murcia Lopez, Jason Drummond, Ye Pan, and David Swapp. 2016. An 'in the wild'experiment on presence and embodiment using consumer virtual reality equipment. IEEE transactions on visualization and computer graphics 22, 4 (2016), 1406-1414.

[40] Diane Watson, Mark Hancock, Regan L Mandryk, and Max Birk. 2013. Deconstructing the touch experience. In Proceedings of the 2013 ACM international conference on Interactive tabletops and surfaces (2013). ACM, New York, NY, USA, 199-208.

[41] Daniel Wigdor and Dennis Wixon. 2011. Brave NUI world: designing natural user interfaces for touch and gesture. Elsevier, New York, NY, USA.

[42] Terry Winograd, Fernando Flores, and Fernando F Flores. 1986. Understanding computers and cognition: A new foundation for design. Intellect Books, Norwood, New Jersey.

[43] Bob G Witmer and Michael J Singer. 1998. Measuring presence in virtual environments: A presence questionnaire. Presence 7, 3 (1998), 225-240. 